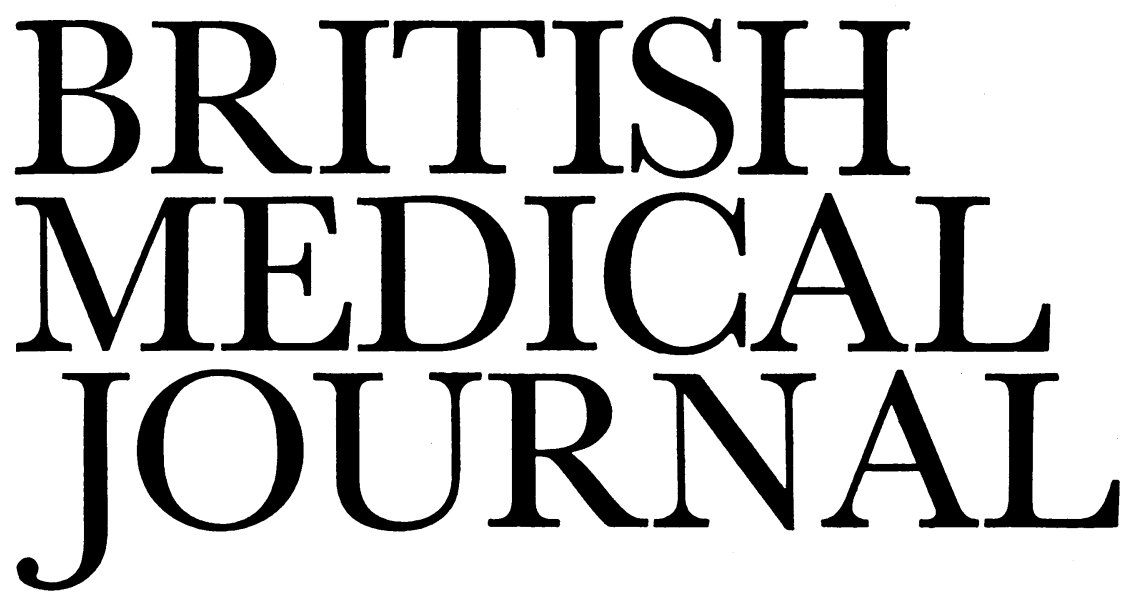

LONDON, SATURDAY 10 JULY 1982

\title{
Perinatal referral: a time for decisions
}

Ill or high-risk newborn babies are commonly transferred from their maternity hospital of birth to one with appropriate neonatal intensive-care facilities. ${ }^{1-3}$ The extent to which individual health authorities have encouraged and funded the development of regional neonatal intensive-care units varies from region to region in Britain. Different patterns of neonatal referral have evolved, partly influenced by the standards of care offered at district maternity hospitals. Some regional neonatal intensive-care units accept premature or low-birthweight babies who are not ill, whereas in other units the referral pattern is dominated by premature babies who need ventilatory support because of impending or actual respiratory failure. Comparison of survival rates and incidences of neurodevelopmental handicap in babies referred to different regional neonatal intensivecare units is unhelpful without detailed knowledge of referral patterns-particularly the proportion of babies with respiratory failure.

The aim of ventilatory support is to keep the baby alive and prevent or minimise neurodevelopmental and other sequelae, while the underlying condition resolves either spontaneously (as in hyaline membrane disease) or with specific treatment. When the baby with respiratory failure is moved by trained staff of the regional neonatal intensive-care unit with ventilatory support facilities he should arrive at the regional unit in at least as good a condition as when he left the referring hospital. Without doubt the referral of babies with. respiratory failure saves lives. Sims and his colleagues ${ }^{4}$ recently reported that the survival rate of babies with respiratory failure who had been declined admission to a regional neonatal intensive-care unit because the unit was full and working to capacity was less than half that of the similar babies fortunate enough to be accommodated. Proving that transfer of premature, high-risk babies without respiratory failure improves their chances of survival is more difficult. Nevertheless, high-quality neonatal care based on anticipation, early recognition, and prompt treatment of complications seems very likely to lessen the risk of both respiratory failure ${ }^{5}$ and neurodevelopmental sequelae. ${ }^{6} 7$

The neonate who requires expert care and is at risk of developing respiratory failure can to a great extent be recognised before birth. Nowadays many regional maternity hospitals accept antenatal referrals of patients with complications such as fetal growth retardation, pre-eclamptic toxaemia, recurrent antepartum haemorrhage, premature labour, and premature rupture of the membranes. ${ }^{89}$ The idea of transferring pregnant women with problems that put their offspring at risk is intellectually attractive; hence the cliche "the safest transport incubator is the womb." Research studies that have pointed to better survival rates of babies transferred antenatally than of those transferred after birth ${ }^{810}$ are, however, of little value-because babies selected for neonatal transfer are generally more ill. A fresh look at antenatal transfer is urgently needed before it spreads by habit. Few babies referred antenatally, about $20 \%$ in our own centre, require definitive ventilatory support. Some obstetricians and paediatricians may consider this to be good antenatal prediction, but the other side of the coin is that $80 \%$ of babies born to referred women take up valuable resources, especially nursing time, so that babies who do require ventilatory support may have to be refused. This problem cannot entirely be solved by transferring mothers and babies back to the referring district maternity hospital as soon as possible.

Might the regional centres play a greater part in the assessment of fetal health in antenatal referrals ?911 Fetal growth retardation, pre-eclamptic toxaemia, and recurrent antepartum haemorrhage, common reasons for referral, may cause asphyxial death even before the onset of labour. Antenatal care cannot reverse these complications of pregnancy; instead the problem essentially resolves itself into the judicious timing of elective preterm delivery to release the fetus from a hostile womb. The benefit of doing this has to be balanced against the risk of exposing the baby to the postnatal hazards of prematurity. Are obstetricians working from regional centres better able to predict impending fetal asphyxia in high-risk pregnancies so that the timing of delivery can be put on a more sound footing ? At present the available diagnostic tools, which include antenatal fetal heart-rate monitoring with or without oxytocin challenge, charting fetal movements, and possibly monitoring fetal breathing movements, lack sharpness. The other common pregnancy complications-spontaneous preterm labour and premature rupture of the membranes-present similar problems. Obstetricians in regional centres are unlikely to be any better at stopping premature labour or preventing or diagnosing fetal sepsis than their colleagues elsewhere.

The benefits that a regional centre offer in high-risk pregnancies arise not so much from the availability of more accurate and advanced fetal diagnostic techniques or fetal therapeutics. 
What the obstetrician does have is the freedom of choice to make decisions when he is confident that the quality of neonatal care will be excellent, however small the baby. This need not and must not be the sole prerogative of obstetricians in regional centres. Even if there were several perinatal centres in each region they would not be likely to cope with all or even most of the high-risk pregnancies presenting at district maternity hospitals. Uncritical expansion of antenatal referral services erodes the experience of medical and nursing staff in the management of common pregnancy complications-yet at the same time accommodation for such patients at the regional centre can never be guaranteed.

There would be little justification for the transfer of premature babies who are not suffering from respiratory failure and for many examples of antenatal referral if the standards of neonatal care in district maternity hospitals were improved. The essential neonatal facilities that must be maintained in district maternity hospitals can be spelt out: constantly available skilled resuscitation and short-term ventilatory support; the ability to anticipate neonatal problems and recognise them early; reliable monitoring for and prophylaxis against apnoeic attacks; scrupulous control of oxygen treatment in babies with respiratory distress; awareness of the individual baby's thermal homoeostasis; and a critical approach to gavage feeding in small babies. The provision of such basic care is not costly, and it is to midwives and nurses we must turn to maintain these standards. An increase in their numbers is certainly necessary in most district maternity hospitals, but ensuring that existing staff are appropriately trained is just as important. Present training programmes, in their attempt to be comprehensive, frequently fail to teach the nuances of neonatal care. Yet in this specialty it is often nuances that dictate the difference between appropriate and poor care.

We have reached a crossroads in the regional organisation of perinatal care in Britain. Our health service administrators must not be allowed to lead us towards too much centralisation in the belief that money can be saved by diverting resources from the district maternity hospitals. What then should be our policy on neonatal and antenatal referral ? Neonatal transfer for impending respiratory failure is totally justified. We need a more selective policy for antenatal transfer that would include, for example, women with fetuses severely affected by rhesus disease; those with complications of pregnancy in whom preterm elective delivery is contemplated in the face of uncertainty about pulmonary maturation or a known risk of hyaline membrane disease based on biochemical analysis of amniotic fluid; and the presence of a fetal malformation amenable to surgical treatment or with a predicted need for medical treatment. Finally, undue emphasis on neonatal and antenatal transfer should not preclude regional perinatal centres from maintaining other roles. Firstly, they should find time to improve standards of care at district maternity hospitals through the training of midwives and nurses and postgraduate medical education; secondly, they need to look at innovations in the prevention and treatment of perinatal disorders-stimulated by close working contact between geneticist, obstetrician, paediatrician, neurodevelopmental specialist, paediatric pathologist, and others; and, thirdly, they should collect and analyse regional and district statistics so that more can be learnt about the influence of different patterns of perinatal care on mortality and neurodevelopmental handicap.

MaLColm L Chiswick

Consultant Paediatrician,

North-western Regional Perinatal Centre,

St Mary's Hospital, Manchester M13 0JH
1 Blake AM, McIntosh N, Reynolds EOR, St Andrew D. Transport of newborn infants for intensive care. $\mathrm{Br} \mathrm{Med} \mathcal{F} 1975$;iv:13-7.

2 Chiswick ML, Davies P, Bate R, Dryburgh E, Gordon-Nesbitt D. Regional organisation of neonatal intensive care. Br Med f 1979;ii:247-50.

${ }^{3}$ Chiswick ML. Regional organisation of neonatal intensive care. In: Wharton B, ed. Topics in perinatal medicine. Tunbridge Wells: Pitman Medical Publications, 1980:120-30.

4 Sims DG, Wynn J, Chiswick ML. Outcome for newborn babies declined admission to a regional neonatal intensive care unit. Arch Dis Child $1982 ; 57: 334-7$.

5 Klaus M, Fanaroff A, eds. Care of the high-risk neonate. Philadelphia: W B Saunders, 1973:119-67.

${ }^{6}$ Pape KE, Fitzhardinge PM. Perinatal damage to the developing brain. In: Milunsky A, Friedman EA, Gluck L, eds. Advances in perinatal medicine. Vol 1. New York: Plenum Medical Book Company, 1981:45-85.

${ }^{7}$ Pape KE, Wigglesworth JS. Haemorrhage, ischaemia and the perinatal brain. London: William Heinemann Medical Books, 1979. (Spastics International Medical Publications.)

${ }^{8}$ Blake AM, Pollitzer MJ, Reynolds EOR. Referral of mothers and infants for intensive care. $\mathrm{Br} \mathrm{Med} \mathcal{F} 1979$;ii:414-6.

${ }^{9}$ Crowley P, Lamont R, Elder MG. The obstetric care of the fetus transferred in utero. F Obstet Gynaecol 1982;2:129-33.

${ }^{10}$ Modanlou HD, Dorchester W, Freeman RK, Rommall C. Perinatal transport to a regional perinatal centre in a metropolitan area. Maternal versus neonatal transport. Am f Obstet Gynecol 1980;138:1157-64.

11 Boehm FH, Haire MF. One-way maternal transport: an evolving concept. Inpatient services. Am $\mathcal{F}$ Obstet Gynecol 1979;134:484-92.

\section{Beta-blockers in the treatment of chronic simple glaucoma}

\begin{abstract}
We ought not to be over anxious to encourage innovation in cases of doubtful improvement, for an old system must ever have advantages over a new one: it is established and understood. C C Colton 1825.
\end{abstract}

A preparation of timolol maleate for topical use was released in Britain in January 1979. By December 1981, 50000 patients with glaucoma were thought to be receiving treatment with this drug. We need, therefore, to review the action of beta-blockers in glaucomatous eyes, to emphasise possible unwanted effects, and to try to identify the place of these drugs in the management of chronic simple glaucoma.

Chronic simple glaucoma is a disease treated in the main by topically applied drugs which lower the intraocular pressure. The side effects on the eye of long-term drug administration include blurred vision, irritation, and pain-and since the disease itself is largely asymptomatic except in the late stages, the side effects discourage patients from taking their drugs regularly. The results are poor control of the intraocular pressure $^{12}$ and progression of the disease. Against that background there has been a great need for a tolerable, effective drug for glaucoma that can be administered topically.

It is now nearly 14 years since the first report of the effect of intravenous and oral propranolol on the intraocular pressure. ${ }^{3}$ This and other beta-blockers have had their ocular hypotensive effect confirmed both when given by mouth and when applied topically. ${ }^{4}$ Not until the advent of timolol, however, did the pharmaceutical industry come up with an acceptable combination of hypotensive effect, duration of action, and relative freedom from local side effects.

Timolol is a beta ${ }_{1}$-adrenergic and beta ${ }_{2}$-adrenergic blocker which has neither any intrinsic sympathomimetic action nor any important local anaesthetic properties. Each $1 \mathrm{ml}$ of $0.25 \%$ solution contains $0.34 \mathrm{mg}$ timolol maleate, and the drug is 